\title{
Impurity-seeded plasma experiments on JET
}

\author{
G.P. Maddison ${ }^{1}$, M. Brix ${ }^{2}$, R. Budny ${ }^{3}$, M. Charlet $^{1}$, I. Coffey ${ }^{1}$, \\ J.G. Cordey ${ }^{1}$, P. Dumortier ${ }^{4}$, S.K. Erents ${ }^{1}$, N.C. Hawkes ${ }^{1}$, \\ M. von Hellermann ${ }^{5}$, D.L. Hillis ${ }^{6}$, J. Hogan ${ }^{6}$, L.D. Horton ${ }^{7}$, \\ L.C. Ingesson ${ }^{5}$, S. Jachmich ${ }^{2}$, G.L. Jackson ${ }^{8}$, A. Kallenbach ${ }^{7}$, \\ H.R. Koslowski ${ }^{2}$, K.D. Lawson ${ }^{1}$, A. Loarte ${ }^{9}$, G.F. Matthews ${ }^{1}$, \\ D. McDonald ${ }^{1}$, G.R. McKee ${ }^{8,10}$, A. Meigs ${ }^{1}$, A.M. Messiaen ${ }^{4}$, \\ F. Milani ${ }^{1}$, P. Monier-Garbet ${ }^{11}$, M. Murakami ${ }^{6,8}$, M.F.F. Nave ${ }^{12}$, \\ J. Ongena ${ }^{4}$, M.E. Puiatti ${ }^{13}$, E. Rachlew ${ }^{14}$, J. Rapp ${ }^{2}$, S. Sharapov ${ }^{1}$, \\ G.M. Staebler ${ }^{8}$, M. Stamp ${ }^{1}$, J.D. Strachan ${ }^{3}$, W. Suttrop ${ }^{7}$, \\ G. Telesca ${ }^{13}$, M.Z. Tokar ${ }^{2}$, B. Unterberg ${ }^{2}$, M. Valisa ${ }^{13}$, \\ K.-D. Zastrow ${ }^{1}$ and EFDA-JET 2000 workprogramme \\ contributors $^{\mathrm{a}}$
}

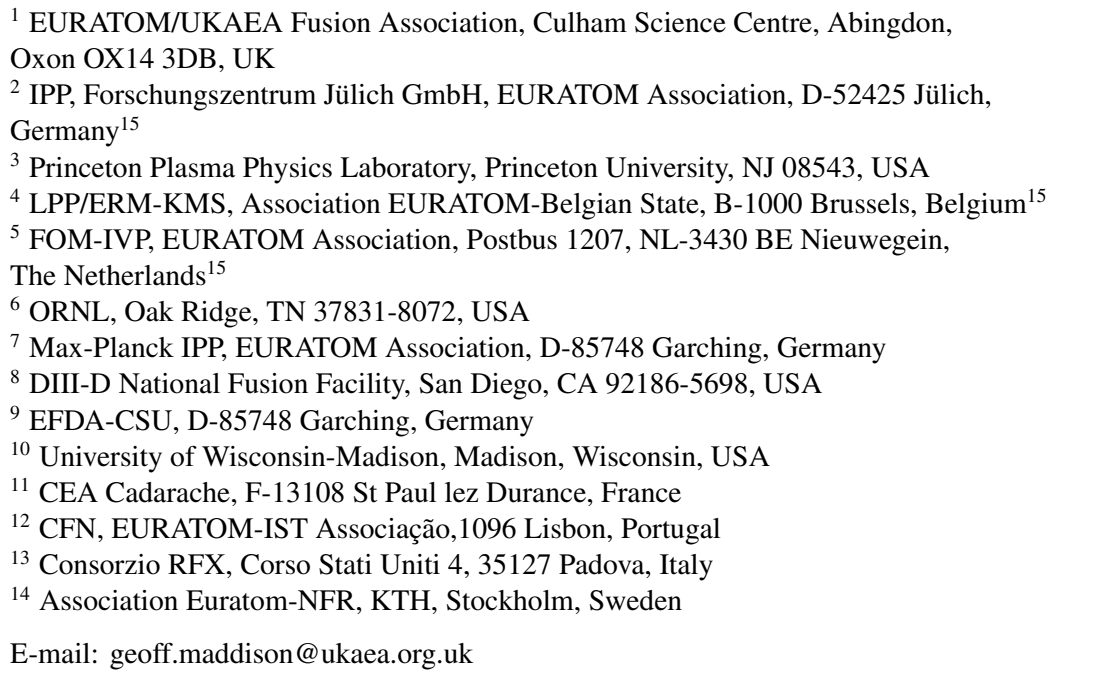

Received 28 January 2002, accepted for publication 21 November 2002

Published 13 December 2002

Online at stacks.iop.org/NF/43/49

\begin{abstract}
Scaling to larger tokamaks of high confinement plasmas with radiating edges, induced by impurities, is being studied through internationally collaborative experiments on JET. In campaigns till the end of 2000, three different regimes have been explored. A small number of limiter L-mode discharges seeded with neon have most closely repeated the approach used on TEXTOR-94, but different collisionality and particle transport in JET impede central peaking of the density associated with improved confinement. Divertor L-modes at intermediate density, again with neon injection, have pursued transiently enhanced states found on DIII-D. Confinement up to H-mode quality, together with radiation fractions of $\approx 40 \%$, have briefly been obtained, though central $Z_{\text {eff }}$ quickly increases. Most effectively, neon and argon seeding of higher density ELMy H-modes formed mainly at low triangularity on the septum of the MkIIGB divertor, resembling a pumped-limiter arrangement, have been examined. Good confinement has been sustained at densities close to the Greenwald level in 'afterpuff' (AP) phases following the end of main gas fuelling, for little change of central $Z_{\text {eff }}$ but up to $\approx 60 \%$ radiation. Outstanding normalized properties up to $H_{97}=0.99$ at $f_{\text {Gwd }}=0.94$ have thus been achieved, above the conventional $\mathrm{H}$-mode density limit for diverted plasmas. Stationarity of states has also been

\footnotetext{
a See appendix of paper by Pamela J 2000 18th IAEA Fusion Energy Conf.

(Sorrento, Italy, October 2000).

${ }^{15}$ Partners in the Trilateral Euregio Cluster (TEC).
} 
extended to many energy confinement times by including low, extra gas inputs in the 'AP', suggestive of an optimized fuelling scheme. Further development in 2001 is reported separately in [1]. Accompanying ELMs are generally reduced in frequency though not evidently in size, electron pedestal pressure being almost unchanged from unseeded behaviour. There are indications of the most favourable impurity species scaling with plasma parameters, performance, radiation and its concentration within a mantle all increasing with argon compared to neon in JET. These benefits in terms of integrated properties are just as required for long burning pulses in ITER, supporting its use of a radiating mantle to assist not only power exhaust but performance too. Impurity-seeded H-modes can therefore contribute directly to next-step scenario development.

PACS numbers: 52.55.-s, 52.55.Fa, 52.55.Rk

\section{Introduction}

Achieving fusion plasma conditions in a next-step tokamak will depend upon maintenance of sufficient confinement, density and core purity, together with acceptable exhaust of power and particles, for many hundreds of energy confinement times. Based upon present experience, the regime which seems best able to approach these aims is the ELMy H-mode, but two principal limitations are still recognized: good confinement measured in terms of scaling laws, e.g. an ITERH97 [2] factor $H_{97} \equiv \tau_{\mathrm{E}} / \tau_{\mathrm{H} 97} \approx 1$, tends to degrade [3] as density is raised close to the Greenwald limit, i.e. for

$$
f_{\text {Gwd }} \equiv \frac{\pi\left\{\bar{n}_{\mathrm{e}} 10^{20} \mathrm{~m}^{-3}\right\}\left\{a^{2} \mathrm{~m}^{2}\right\}}{\left\{I_{\mathrm{p}} \mathrm{MA}\right\}} \rightarrow 1 ;
$$

adequate confinement also tends to be accompanied by low frequency, but large Type I ELM fluctuations which impose severe transient loads on intercepted surfaces [4], reducing their endurance and possibly releasing impurities into the plasma. To mitigate the latter, a number of devices have tested deliberate addition of intermediate atomic number impurities, mainly noble gases, at the plasma edge, in order to increase the fraction of efflux power dispersed over greater surface areas as mantle radiation. This led in the ASDEX Upgrade (AUG) divertor machine to the so-called 'completely detached H' (CDH) mode [5], which had $H_{97} f_{\text {Gwd }} \sim 1$ without any ELMs penetrating through to target surfaces. However, central $Z_{\text {eff }}$ was raised appreciably, and efforts to reproduce the regime in JET were unable to avoid strong impurity penetration and declines in confinement. One discriminating factor may have been the larger ratio of plasma to divertor volume in JET than for AUG. On the other hand, concurrent tests in the TEXTOR-94 pumped-limiter tokamak separately observed not only that high radiation fraction $f_{\text {rad }} \equiv P_{\text {rad }} / P_{\text {in }}$ could be obtained without significantly affecting core $Z_{\text {eff }}$, but that for high enough values $f_{\mathrm{Gwd}}, f_{\mathrm{rad}} \geqslant 0.7-0.8$, a spontaneous transition to an ELM-free state with improved confinement, up to H-mode level, actually occurred [6]. This so-called 'radiative improved' (RI) mode [6,7], reminiscent of earlier observations in the smaller ISX-B device [8], also exhibited extremely long stationarity, and recovered the linear 'neo-Alcator' scaling of energy confinement with density seen in Ohmic plasmas (LOC), but now with auxiliary heating and above the usual Shimamura point at which saturation of the former characteristics intervenes (SOC) [7]. This alternative scheme therefore offered the potential to realize all requirements of a next-step state simultaneously. A vital next question then became its possible scaling to larger systems, requiring study of its reproducibility, e.g. on JET and JT-60U [9].

During 1999 and 2000, preliminary efforts were made to replicate the discovery conditions of TEXTOR-94 as closely as possible in JET by injecting impurities into neutral beam heated $(\mathrm{NBH})$ L-modes formed against its discrete outboard limiters [10-12]. However, their relatively poorer conditioning and power handling have so far restricted the number of such experiments [10]. First confinement gains from impurity 'seeding' of diverted plasmas were observed transiently in L-modes using single-null geometry with NBH on the DIII-D tokamak [13], where injection of neon during early relaxation of the current density profile produced enhancements up to $\mathrm{H}$-mode level whilst retaining the advantageous L-mode edge. Importantly, lower normalized densities $f_{\mathrm{Gwd}} \approx 0.2-0.4$ were involved as well. Attention has also been given to similar conditions in JET single-null discharges [12, 14, 15], using its conventional vertical targets equilibrium within the MkIIGB gas-box divertor [16], specifically to study the physics of impurity effects. Beyond these more basic tests, though, most interest has been focused on novel trials of impurity puffing into higher density ELMy H-mode plasmas in JET $[12,17]$, to resume moderation of their difficult features while perhaps combining with the benefits of RI-modes, and so continue development of this promising next-step regime. Both magnetic equilibria used and seed species added have been varied. In particular, for low plasma triangularity, so-called 'afterpuff' (AP) stages following the end of main gas input have been adapted to obtain steady high-performance phases effectively pointing towards an optimized fuelling and power radiation strategy. Each of these areas of activity up to the end of 2000 is reviewed, further work in 2001 being described in [1] together with additional details of confinement in seeded H-modes in [18]. Finally, a summary is presented, before discussing points of continuing investigation and considering the prospects for application to the ITER next-step device.

\section{Limiter plasmas}

Steady RI-modes have been pioneered and most extensively studied in circular, pumped-limiter plasmas in TEXTOR-94 $[6,7]$, chiefly beginning from L-modes involving at least $\approx 20 \%$ heating by neutral beams, then with impurities added reaching confinement of $\mathrm{H}$-mode quality for high radiated power fraction $f_{\text {rad }} \geqslant 0.8$ and densities around or 
even exceeding [19] Greenwald level. The most closely related configuration in JET, although with much weaker wall and target-surface pumping only, involves moderately elongated $(\kappa \approx 1.4)$ plasmas resting against its 12 discrete outboard carbon limiters [11] (see figure 1 $(a)$ ). Partly to assist power handling on these elements, $B_{0}=2.9 \mathrm{~T}, I_{\mathrm{p}}=1.8 \mathrm{MA}$ yielding $q_{a} \approx 5$, i.e. shallower field-line incidence, were chosen. No direct fuelling other than from the neutral beams during heating, and the short neon puffs $(\mathrm{P})$ applied, was required, consistent with a low pumping state. Waveforms for a typical matched pair of discharges without and with impurity seeding are contrasted in figure 2 . Radiated power can be seen to rise steadily after neon injection, but central lineaverage $Z_{\text {eff }}$ and soft x-ray (SXR) emission show similarly prompt increases suggesting its rapid permeation across the entire plasma. Formation of a MARFE at the inboard side is indicated at $21.2 \mathrm{~s}$ later in this pulse by its sudden rise in the horizontal $\mathrm{D} \alpha$ signal.

Auxiliary heating was varied up to $8 \mathrm{MW}$ of NB power over the set of neon experiments, and values of $f_{\mathrm{Gwd}}$ up to $\approx 80 \%, f_{\text {rad }}$ up to $\approx 70 \%$, for central line-average $Z_{\text {eff }} \leqslant 4$, were reached. As exemplified in figure 2, however, marked gains in confinement like those in TEXTOR-94 were not yet observed. Generally, confinement was only slightly increased relative to divertor L-mode performance measured by the ITERH89P scaling law [20] $H_{89} \equiv \tau_{\mathrm{E}} / \tau_{\mathrm{H} 89 \mathrm{P}}$, viz $H_{89} \approx 1.3$, and even this normalized quantity tended to decline with increasing input power. Variation of central $Z_{\text {eff }}$ with radiated power fraction was analysed using full tomographic reconstruction of its poloidal distribution from multiple bolometric lines-of-sight (LsOS). In addition to conditions being restricted by appearance of lower inboard MARFEs, again as demonstrated in figure 2, a small region of strong emission also tended to arise in the upper inboard position. It remains uncertain without full toroidal coverage by diagnostics whether the latter is axisymmetric, but it is still probably due to limiter and/or local wall tile interactions,
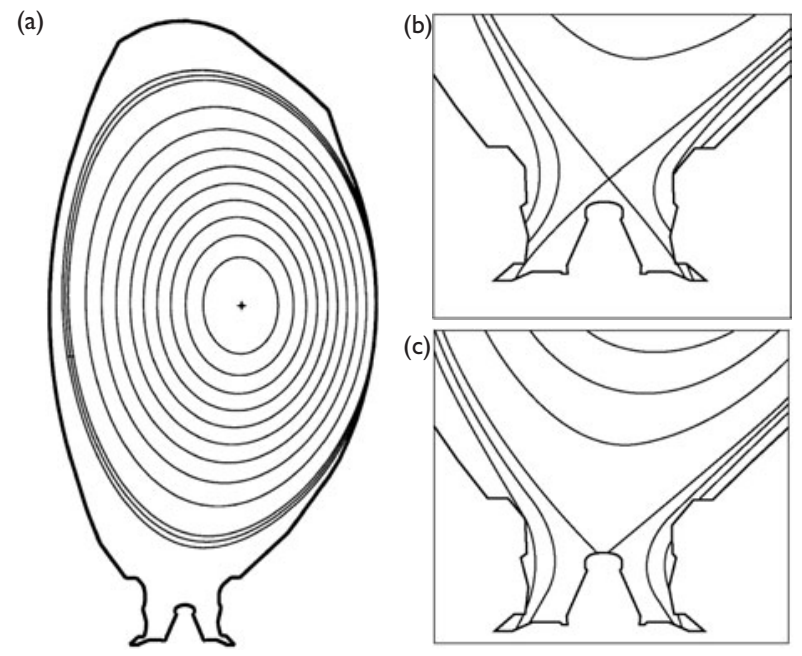

Figure 1. Representative magnetic equilibria within JET MkIIGB geometry used in each set of trials: $(a)$ outboard limiter; $(b) \mathrm{X}$-point, with strike-points and divertor SOL intercepting the side ('vertical') target plates ('corner' configuration is obtained by lowering the strike-points so the SOL reaches the bottom corners of the divertor); (c) 'septum', resembling a pumped-limiter arrangement. consistent with spectroscopic observations that carbon always remained the dominant impurity [11]. Allowing for this top inboard emission, total radiated power in fact conforms well with the Matthews scaling [4], as illustrated in figure 3 by comparing measured with predicted $Z_{\text {eff }}$ values. Hence, impurity behaviour still resembled that prevailing in a range of other experiments, as embodied in the Matthews relation.

A defining feature of RI-modes in TEXTOR-94 is a strong correlation of their improved confinement with accentuated central peaking of the density profile [7,21], a central to volume-average ratio $n_{\mathrm{e}}(0) /\left\langle n_{\mathrm{e}}\right\rangle_{\mathrm{vol}} \approx 2.6$ being typical at similar $q_{a} \approx 5$. A key distinction was that conversely density profiles in JET tended to remain comparatively flat, $n_{\mathrm{e}}(0) /\left\langle n_{\mathrm{e}}\right\rangle_{\text {vol }} \leqslant 1.6$. Two preliminary attempts to increase peaking by inboard injection of frozen deuterium pellets did not alter this situation. Possible impact of differing source distributions between the two devices has been tested using the RITM self-consistent particle and heat transport code [22, 11], firstly by adjusting shaping factor $s \equiv-\left(v^{\text {pinch }} / D_{\perp}\right)\left(a^{2} / r\right) / q$, where $D_{\perp}$ and $v^{\text {pinch }}$ are the particle diffusivity and inward pinch velocity $(<0)$, so as to match the JET profiles in a typical neon-seeded limiter case with $f_{\text {rad }} \approx 0.7$ (yielding $s \approx 0.5$ ). Then keeping this identical $s$ but taking full account of the different source distribution due to fuelling and added impurities, profiles have been computed for an RI shot in TEXTOR-94 at similar $f_{\text {rad }}$ and $q_{a}$. In contradiction to its actual experimental peaking of $n_{\mathrm{e}}(0) /\left\langle n_{\mathrm{e}}\right\rangle \approx 2.5$, this procedure would imply a rather flat density profile also for TEXTOR-94, similar to the JET shape [11]. More appropriate shaping factors for the former are actually found [23] to be around $s \approx 3\left(\left(a^{\mathrm{TEXTOR}} / a^{\mathrm{JET}}\right) \approx 0.5\right)$. In other words, contrasting sources alone cannot account for the real difference

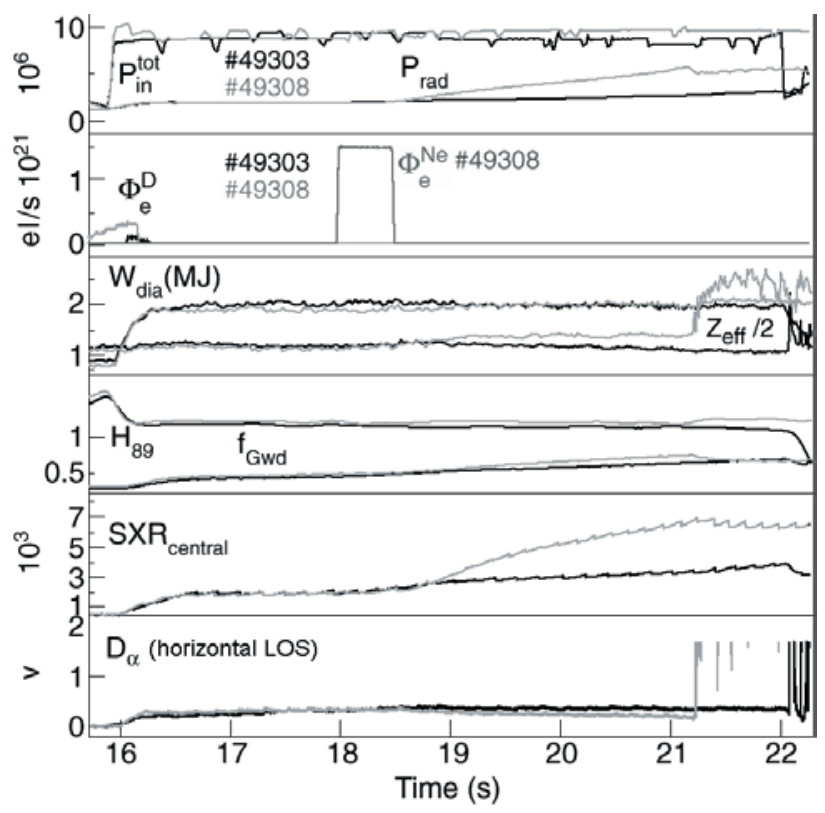

Figure 2. Time traces for typical outboard limiter pulses without (\#49303) and with (\#49308) neon seeding: total input (mainly NBH) and radiated power; gas fuelling rates; diamagnetic stored energy and central line-average $Z_{\text {eff }}$; confinement normalized to ITERH89P L-mode scaling and Greenwald density fraction; central SXR emission; D $\alpha$ emission along a horizontal LOS. The sudden rise at $21.2 \mathrm{~s}$ in \#49308 signifies condensation of a lower inboard MARFE. 


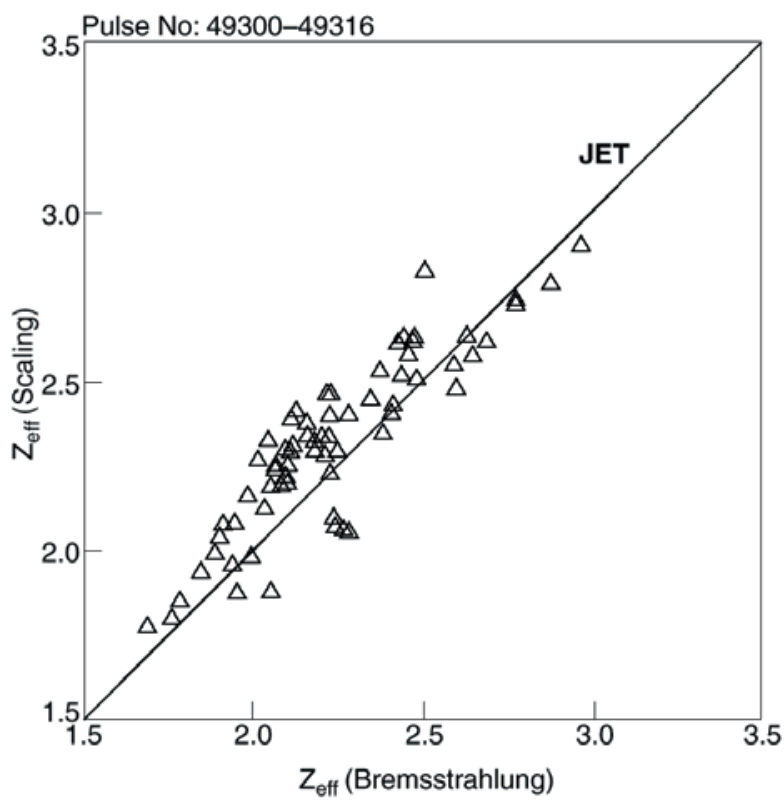

Figure 3. Comparison for JET outboard limiter shots with neon of central line-average $Z_{\text {eff }}$ measured by bremsstrahlung with that predicted by the Matthews scaling using tomographic reconstruction of total radiated power. (Points plotted at multiple times in each pulse.)

in density peaking on JET, and distinct underlying transport ( $v^{\text {pinch }} / D_{\perp}$ ) does seem to be involved.

Current understanding of cross-field fluxes in moderately collisional regimes proposes that ion temperature gradient (ITG) instabilities dominate in L-modes, resulting in a flat density profile, but when they are quenched, dissipative trapped electron modes (DTEMs) alternatively can produce a relatively strong inward particle pinch and consequently increased central peaking [24]. The interplay in the absence of strong rotational shear may be expressed [24] in terms of an integral steady-state continuity equation $\Gamma_{\mathrm{r}}-(1 / r) \int_{0}^{r} S_{\mathrm{e}} r \mathrm{~d} r \equiv$ $G(\rho)=0$, where $S_{\text {e }}$ are particle sources and $G$ is a governing function of the ratio of gradient scale-lengths $\rho \equiv L_{\mathrm{T}} / L_{\mathrm{n}}=$ $1 / \eta_{\mathrm{i}}$. During L-mode, at an intermediate radius where the change in density gradients is greatest, large ITG contributions lead to a folded form for $G$ with two stable points, but only the lower, flatter one with higher turbulence is accessible (see figure 4). However, as impurities are added, suppression of ITG growth rates by rising $Z_{\text {eff }}$ lowers the intervening maximum value of $G$, until beyond a certain critical position it falls below zero, making a bifurcation to the now accessible upper stable point possible. The resulting transition to a more peaked density state with then strongly reduced ITG components is quantitatively well correlated with the entry to RI-mode in TEXTOR-94, supporting this interpretation of its mechanism of improved confinement [11,24]. An equivalent calculation for $G(\rho)$ at a similar normalized radius during neon seeding of a JET limiter plasma is depicted $[11,12]$ in figure 4. It is revealed at once that it remains above the critical form of $G$, with persisting ITG transport, so denoting a flat, low $\rho$ density distribution just as detected in the experiment. Note DTE modes and inward pinching are themselves less apt to increase at the lower collisionality exemplified [25]. Also superimposed in figure 4 are calculated impacts of either

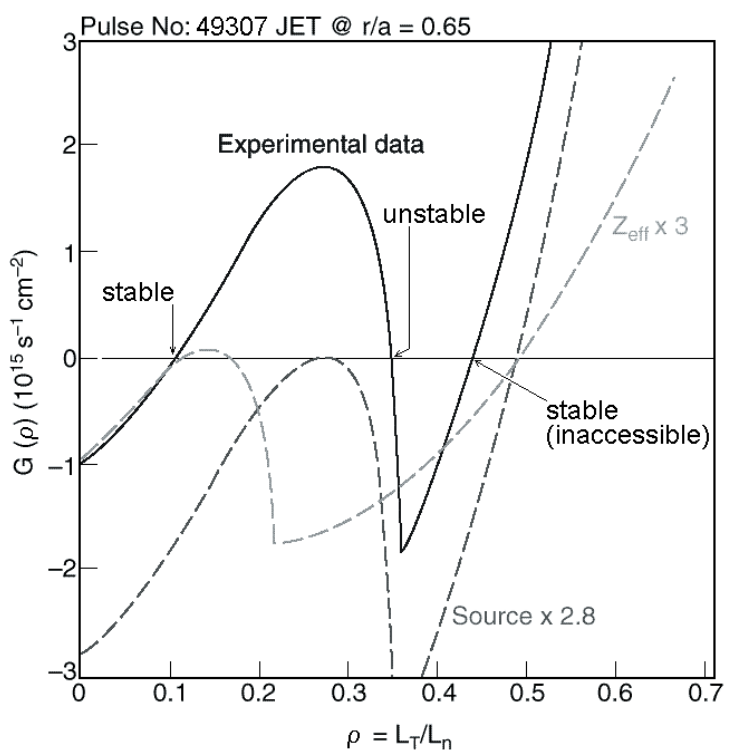

Figure 4. Calculated peaking function $G$ during neon seeding into a JET outboard limiter plasma, from the difference between particle efflux and its integrated source distribution. Roots at $G=0$ determine profile peaking $\rho$; here the upper RI-like solution remains inaccessible, in agreement with observations. Superimposed calculations (- - - ) suggest roughly threefold increases of either $Z_{\text {eff }}$ or interior particle sources separately would be needed to admit a transition to higher $\rho$.

higher $Z_{\text {eff }}$ or a stronger interior particle source, each of which separately would require roughly a threefold increase to reach the critical $G$ and so effect an equivalent transition to RI-mode in JET. The former recourse is clearly less attractive, while the latter remedy ought best to avoid higher central temperatures at the same time, e.g. accompanying strengthened central NBH, since these would tend to exacerbate ITG growth. Further development of deep pellet injection could therefore be appropriate. Lower collisionality [25] and lack of sufficient density peaking yet in JET limiter plasmas thus seem to have been key factors in their inability to access RI-modes most like those in TEXTOR-94.

\section{Divertor L-modes}

In DIII-D single-null diverted plasmas mainly with NBH, neon has been injected prior to any transition into $\mathrm{H}$-mode and before the onset of sawteeth $[13,15]$, deferred by using early beam injection and higher $q_{95} \approx 5$. Improvements in confinement up to H-mode level have been seen, while retaining ELM-free L-mode edge properties. The gains are generally transient, typically being arrested by emergence of sawteeth or growth of neo-classical tearing modes (NTMs) [15], although then $H_{89}$ can sometimes remain significantly above unity. Importantly too they occur at lower normalized density, $f_{\mathrm{Gwd}} \approx 0.4$ or even less, together with moderate radiation fractions $f_{\text {rad }} \approx 0.6$. Similar intermediate density L-mode conditions have been pursued in JET using the standard vertical targets configuration of its MkIIGB divertor [16] (see figure 1(b)), as an opportunity particularly to study impurity effects in divertor geometry. A representative discharge scheme at $B_{0}=2.2 \mathrm{~T}, I_{\mathrm{p}}=1.7 \mathrm{MA}$ with ramped heating and early seeding of neon is illustrated 
in figure 5, emphasizing its initial interval of strongest profile development. During this phase, divertor $\mathrm{D} \alpha$ emission remains low and irregular, but normalized confinement does rise briefly to $H_{89} \approx 2$ while $f_{\text {rad }}, f_{\text {Gwd }} \approx 0.4$. Similar plasmas without seeding tend to enter $\mathrm{H}$-mode, but the neon is consequently leading transiently to comparable confinement while cooling the edge enough to prevent any ELMs [26]. As for JET limiter plasmas above, line-average $Z_{\text {eff }}$ and central SXR emission both rise promptly as well in figure 5, again suggesting rapid intrusion of neon into the core (though mounting heating may also be contributing to the SXR increase). Impurity density profiles from charge-exchange recombination spectroscopy (CXRS) in other instances confirm such penetration and its persistence after neon puffing ceases $[12,14]$. In fact, indications of significant fuelling from injected neon, and relatively slow decline of radiation and $Z_{\text {eff }}$ after its input ends, plus a tendency for $Z_{\text {eff }}$ to increase with higher $f_{\text {rad }}$, all point to only weak divertor control of impurities in these experiments [12]. For the pulse in figure 5, peaking of $H_{89}$ occurs for lower power and subsides before the steady heating phase is reached, actually rolling over around the onset of an $n=2$ MHD mode, followed shortly by the start of sawteeth (note the strike-points are raised too in the pulse shown, settling at their higher position from the dashed arrow onwards). Sawteeth themselves in these JET discharges generally remain small enough at lower input power not to stimulate MHD activity, or to feature directly in confinement decreases. At higher power, addition of ICR radio frequency heating, as in the case shown, has not yet produced any significant delay in their onset, despite Alfvén magnetic signatures of fast particle populations appearing. Pre-heating with lower hybrid waves (LHCD) was

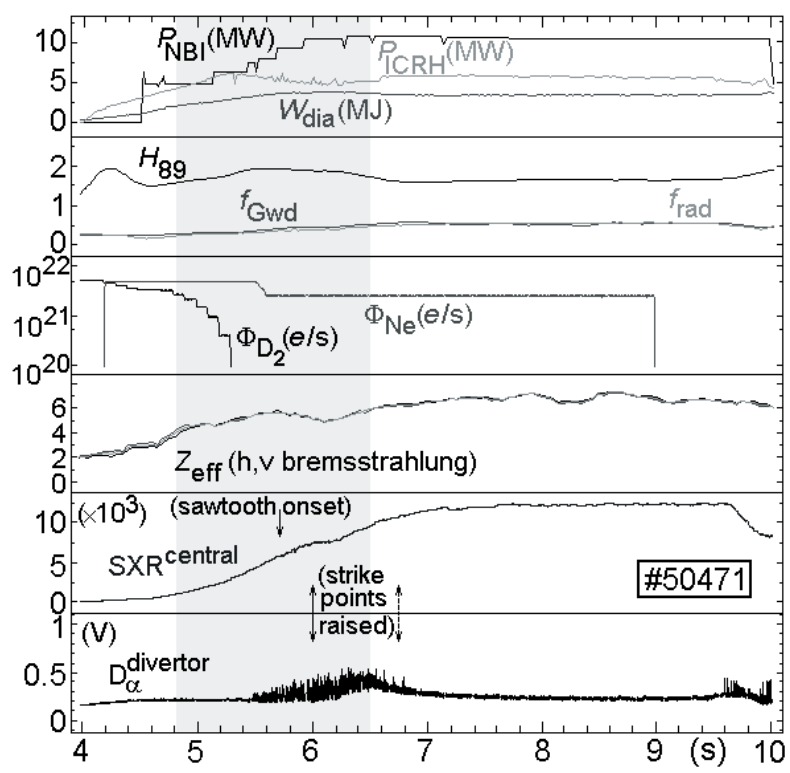

Figure 5. Time traces for a sample divertor L-mode discharge with neon seeding, highlighting the early interval of profile development and transient peaking of normalized confinement: auxiliary heating power and diamagnetic stored energy; confinement normalized to ITERH89P, Greenwald density and radiated power fractions; gas fuelling rates; line-average $Z_{\text {eff }}$ from horizontal and vertical LsOS; central SXR emission; divertor D $\alpha$ emission. The strike-points are raised from the time of the first vertical arrows, holding their new positions from the second arrows onwards. more successful in averting sawteeth, but large rotating loworder MHD modes and roll-over of confinement still tended to occur. Hence the current density profile itself seems more susceptible to performance-limiting instabilities, and could be further optimized [26]. Internal inductance $\ell_{\mathrm{i}}$, for instance, is raised by impurities in JET, but is not instrumental in confinement improvements in DIII-D [10].

A summary over scans of toroidal field strength, input power, and a few plasmas seeded with argon as well as the main set with neon, is presented in figure 6. One point is plotted for each pulse, at a time of maximum properties, whether before or after sawteeth begin. There is little indication of any confinement dependence on magnetic field strength, best results emerging rather at higher Greenwald fractions, and above all for higher input power at moderate field. Two of these latter JET pulses achieve $H_{89}, f_{\mathrm{Gwd}}$ factors and durations quite close to those of DIII-D experiments, as revealed by superimposing respective traces in dimensionless time [15], normalizing each by its mean value of $\tau_{\mathrm{H} 89 \mathrm{P}}$ from the start of seeding to the latest time drawn (figure 7). Note a DIII-D example is given [15] where best performance is interrupted by NTM activity, as indicated by the magnetic signal included. In the JET example properties again reach an initial peak, but then sustain almost these high levels for an extended period (no $n=2$ mode), during which incipient low frequency ELMs develop with amplitudes in terms of total stored energy drops $(\Delta W / W) \approx 5 \%$ only or less. Hence as commented, neon puffing seems capable of moderating ELMs in tentative $\mathrm{H}$-mode states through intensified edge radiation. A notable feature in figure 7 is that while neon input per unit volume is larger in JET, its rise of radiated power is much slower than in DIII-D, although a larger part of the DIII-D seeding is here applied more impulsively. Similar $H_{89}$ and $f_{\mathrm{Gwd}}$ are thus achieved for lower $f_{\text {rad }}$ in JET. Another important result is that while $Z_{\text {eff }}$ is rising to $\approx 5$ in the initial peaking stage, close to typical values of 3-3.5 in DIII-D [15, 26], nevertheless

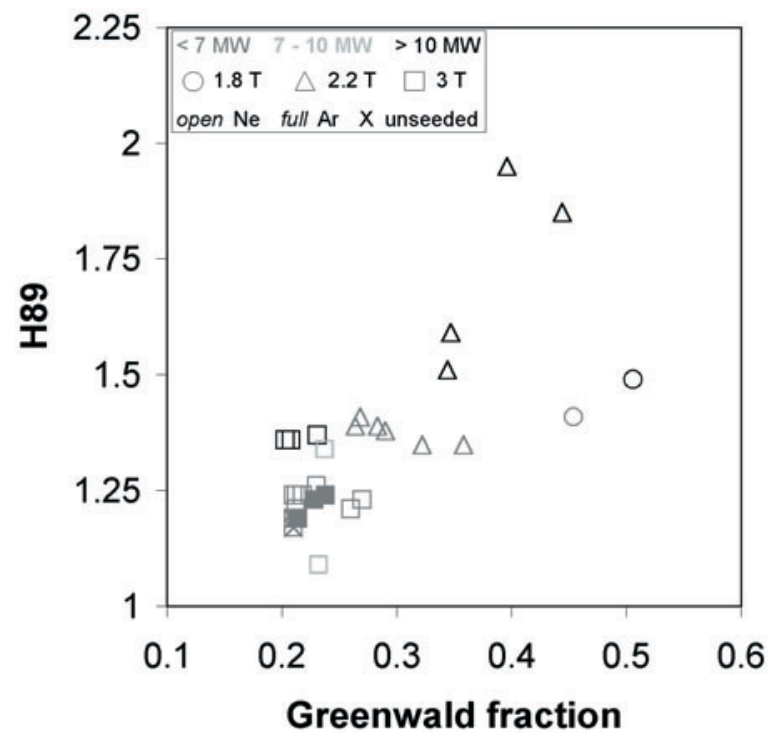

Figure 6. Normalized confinement versus Greenwald density fraction for JET divertor L-modes (one point per shot). Different toroidal field strengths, heating power levels, and seed species, are discriminated. 


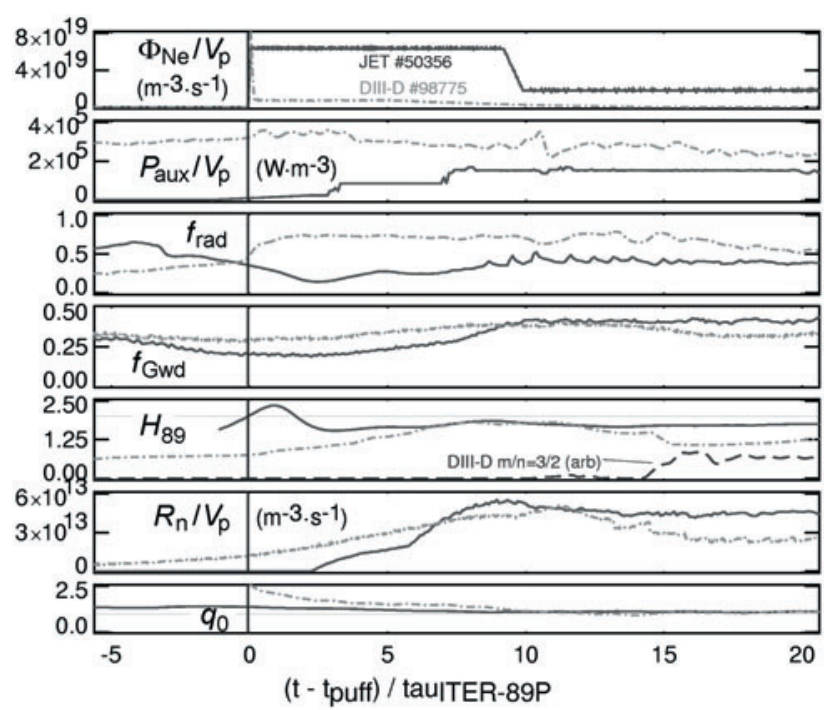

Figure 7. Neon-seeded divertor L-modes in JET (\#50356) and DIII-D (\#98775), contrasting traces over time normalized by mean energy confinement time according to ITERH89P scaling, following the start of neon input: neon puffing per unit plasma volume; heating power per unit plasma volume; radiated power fraction; Greenwald density fraction; ITERH89P confinement factor (inset for DIII-D: magnetic signal showing the rise of a NTM); neutron emission per unit plasma volume; central safety factor. Note the slower rise of $f_{\text {rad }}$ in JET despite its higher seeding rate.

the volumetric neutron production rate is also monotonically increasing, denoting a corresponding improvement in core plasma quality. Subsequent JET experiments have in fact established a doubling of central ion temperature and of the thermal neutron rate [26]. This relates to a key point for impurity seeding, since it should be recalled that for a single minority species of charge $Z_{\text {imp }}$ then majority concentration $n_{\mathrm{D}} / n_{\mathrm{e}}=\left(Z_{\mathrm{imp}}-Z_{\text {eff }}\right) /\left(Z_{\text {imp }}-1\right)$, i.e. for a fixed $Z_{\mathrm{eff}}$, higher atomic number impurities are less diluting. Introduction of heavier radiating species can therefore tolerate somewhat higher $Z_{\text {eff }}$ without compromising fuel abundance, and can remain compatible with fusion plasmas.

In contrast to TEXTOR-94 RI-modes, DIII-D seeded L-modes tend not to exhibit strong density peaking [14], and for the JET cases in figure 6 highest confinement factors indeed occurred for low values of $n_{\mathrm{e}}(0) /\left\langle n_{\mathrm{e}}\right\rangle \approx 1.6-1.9$, identical to the unseeded reference plasmas. Analyses with the TRANSP local transport code for a shot pair without and with neon at $3 \mathrm{~T}$, so having a higher $\mathrm{H}$-mode threshold, disclose a clear decrease of ion thermal diffusivity in the outer region of the plasma almost immediately seeding starts, followed after a short delay by a similar relative decline farther inwards $[10,14]$ (figure 8 ). Increases in energy stored within each radius are approximately correlated with the respective falls, while neutron emission again grows throughout the neon puffing, at the end of which $\chi_{\mathrm{i}}$ is considerably reduced over the whole plasma cross-section. At the same time, LIDAR Thomson scattering measurements of electron temperature profiles display greater central peaking with than without neon, presumably owing to radiative cooling of the edge, but otherwise reveal no systematic perturbation of $T_{\mathrm{e}}$ as impurity radiation builds up [10]. Hence there is no evidence of a merely gyro-Bohm like reduction of $\chi_{\mathrm{i}}$ due to lower temperatures, and a clear improvement at

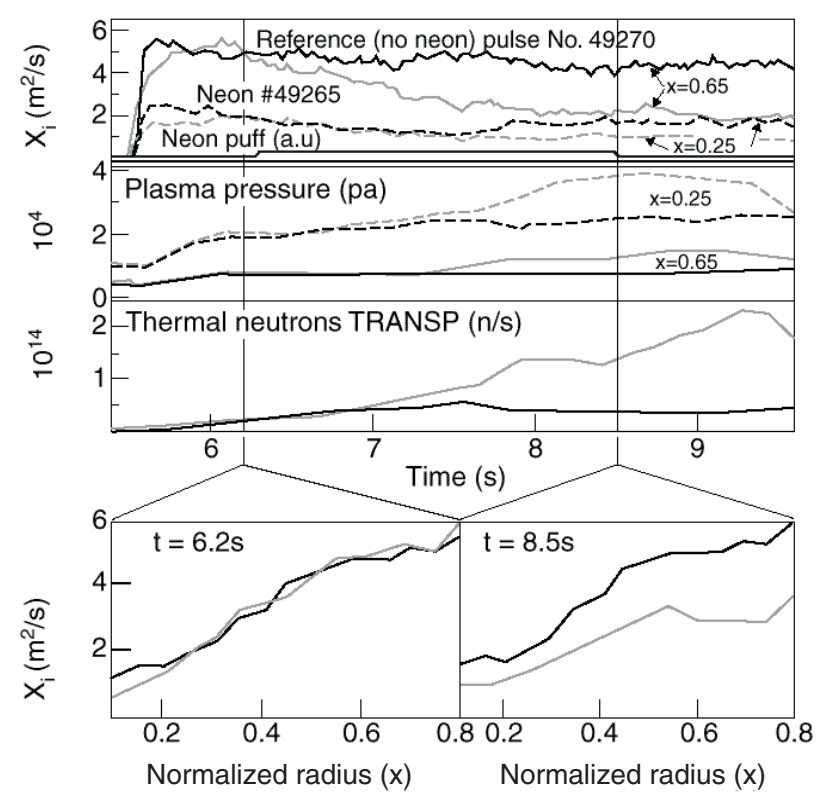

Figure 8. Local analyses with the TRANSP code of ion thermal diffusivity $\chi_{\mathrm{i}}$ and plasma pressure at two normalized radii, plus thermal neutron emission, for a pair of JET divertor L-mode pulses at $3 \mathrm{~T}$ without (\#49270) and with (\#49265) neon injection. Inset are respective profiles of $\chi_{i}$ at two times, showing ion transport decreases over the whole plasma cross-section.

least in ion transport does seem to be implied. Further modelling [25] with the RITM code indicates ITG modes tend to be suppressed directly by increased $Z_{\text {eff }}$ following neon injection, contributing to decreased transport even without density peaking. Moreover, DTEM instabilities tend to be amplified and assume highest normalized growth rates in the outer part of the plasma, potentially reinforcing an inward particle pinch. Similar features are also calculated by the GKS gyro-kinetic code [14], which on the other hand suggests that benefits of stabilizing ITG components are partially offset by replacement with DTEM energy transport in the edge, until these modes gradually decline away as the neon is pumped out. This shift may help explain the modest level of overall confinement generally ( $\mathrm{cf}$ figure 6 at $3 \mathrm{~T}$ ) in spite of the lessening of $\chi_{i}$. In fact, the balance between density peaking and enhanced confinement seems to be a delicate one for these conditions: highest $H_{89}=1.95$ in figure 6 (\#50471) occurs for lower $n_{\mathrm{e}}(0) /\left\langle n_{\mathrm{e}}\right\rangle \approx 1.9$, consistent with smaller ITG fluxes but still only a single, low peaking root of continuity relation [24] $G\left(L_{\mathrm{T}} / L_{\mathrm{n}}\right)=0$ introduced in section 2; whereas higher $n_{\mathrm{e}}(0) /\left\langle n_{\mathrm{e}}\right\rangle \approx 2.3$ correspondingly emerges [25] (\#50329) only at lower $H_{89}=1.5$ (lowest black triangle in figure 6). An alternative candidate for the mechanism of turbulence stabilization in radiatively enhanced performance is strongly sheared plasma rotation [27], especially with unbalanced NBH as applied in JET. In the highest $H_{89}$ plasmas at $2.2 \mathrm{~T}$ (figure 6), however, CXRS detection of toroidal rotation again sees no sharp gradients or temporal developments of the angular velocity, so within the uncertainties, concentrated shear seems not to be evident at least in this component of the motion. Conversely, an indication of accelerated central rotation and shear stabilization over parts of the plasma has been seen in the later L-mode seeding experiments [26] mentioned above. 
Similar unsteady increases of confinement to those found in DIII-D thus have been produced by adding neon in JET MkIIGB divertor L-modes, but the actual processes involved have yet to be clearly resolved and remain under study for such lower collisionality states. Rapid changes in toroidal rotational shear believed to contribute to turbulence reduction $[27,28]$ in particular are considered more fully elsewhere [26].

\section{ELMy H-modes}

As mentioned above, a next-step plasma will require good values of many properties simultaneously, and the key measure of an experiment is accordingly its integrated performance. There is consequently a strong incentive to try to combine into ELMy H-modes some of the benefits found for radiative mantle plasmas. With the JET MkIIGB divertor, a novel arrangement became available by lowering the magnetic X-point onto or just inside the domed barrier dividing its inboard and outboard sides (see figure 1 $(c)$ ), yielding the so-called 'septum' configuration. This geometry has greater width for the scrapeoff layer (SOL) while distancing much recycling from the cryopump, resembling somewhat a pumped-limiter system. It was observed early in the study of septum plasmas both that their ELM characteristics tended to differ from usual divertor behaviour, and that their power threshold for entry into $\mathrm{H}$-mode was substantially lower, although the reason has yet to be determined [29]. An adjunct, however, is that they may have a greater capacity for dispersing power as radiation without falling back into L-mode, and therefore impurity seeding of $\mathrm{H}$-modes has been concentrated initially on this configuration. First trials also focused on neon or argon injection during the phase of main gas fuelling to higher density [17], but it is known that the latter puffing itself generally degrades confinement, so contributing to the conventional $\mathrm{H}$-mode density limit [3]. Conversely, confinement tends naturally to recover again after gas input ends (whether or not impurities are admitted), defining a so-called 'AP' stage of favourable performance. Attention has thus been turned to lengthening AP phases of JET H-mode discharges, progressing from no gas fuelling at all to determine the so-called 'natural' state, i.e. the density from just surface and NB sources, to preceding deuterium fuelling only, then adding neon or argon. Impurities have been injected both during the period of main puffing to reach high density, and later also as smaller inputs afterwards to seek more stationary conditions. Septum pulses at low triangularity (upper value $\delta^{\mathrm{u}}=0.18$ ) have been contrasted with ones having higher shaping ( $\delta^{\mathrm{u}}=0.33$ ) since this can modify ELMs too. In addition, sample diverted plasmas have been included, positioning the strike-points either on the MkIIGB vertical side targets, or in the bottom corners of its structure (see figure $1(b)$ ), in order successively to raise pumping and help expose its influence. Such cases are eventually most important, of course, since improved schemes for divertor machines, like the ITER-FEAT design, are finally being sought.

Signals for a representative pair of septum $\mathrm{H}$-modes at $B_{0}=2.4 \mathrm{~T}, I_{\mathrm{p}}=2.5 \mathrm{MA}$ without and with impurity seeding are contrasted in figure 9 (illustrating the single instance so far in which low argon influx during main deuterium puffing was combined with even lower neon influx immediately afterwards [12]). In both cases, as noted above, stored energy

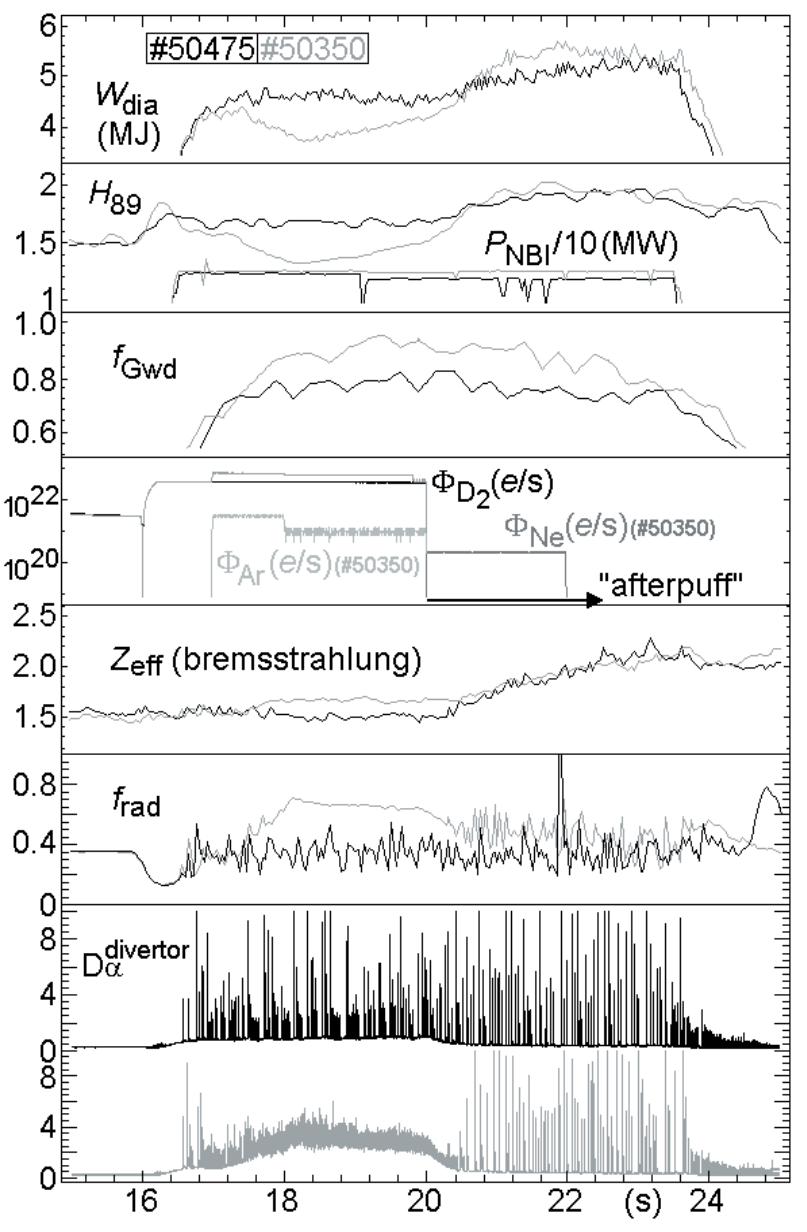

Figure 9. Time traces for a pair of septum H-modes without (\#50475) and with (\#50350) impurity seeding: diamagnetic stored energy; normalized confinement and NB heating; Greenwald density fraction; gas fuelling rates; line-average $Z_{\text {eff }}$; radiated power fraction; divertor $\mathrm{D} \alpha$ emission. The stage following the end of main $\mathrm{D}_{2}$ puffing is designated the 'AP', during which confinement recovers to good H-mode quality. With impurity seeding higher density is maintained as well, for increased radiation but no relative change in $Z_{\text {eff }}$.

and normalized confinement e.g. in terms of $H_{89}$ return to good H-mode quality in the AP, but when impurities are introduced higher density is maintained as well. This aptitude to increase density, and to forestall its decline seen in the AP without seeding, results in an outstanding combination of confinement factor and Greenwald fraction beyond the usual $\mathrm{H}$-mode density limit. In effect, particle confinement is improved and/or the usual degradation of fuelling efficiency for $f_{\mathrm{Gwd}} \rightarrow 1$ which underlies the density limit seems to be lessened. While not so far recovering definitive RI-mode transitions as found in TEXTOR-94, therefore, seeding of $\mathrm{H}$-modes in JET has equivalently revealed a crucial benefit in terms of integrated properties. At the same time, ELMs are typically slowed or diminished, the radiated power fraction is increased, while plasma purity indicated by central lineaverage $Z_{\text {eff }}$ is little altered from the unseeded reference behaviour. Values up to $f_{\text {rad }} \approx 0.6$ have been obtained in septum APs with argon described here for approximately no relative change in $Z_{\text {eff }}$ (see figure 11). Maximum properties in figure 9 are sustained for a time $\approx 3 \tau_{\mathrm{E}}$, during 
which calculations with TRANSP do determine significantly decreased thermal diffusivities for both ions, and the effective coefficient describing total transport, across most of the plasma cross-section. In addition, some modest density peaking is seen (\#50350), though $n_{\mathrm{e}}(0) /\left\langle n_{\mathrm{e}}\right\rangle<1.6$ actually prevails in every example presented. Stonger central peaking has subsequently been observed in an AP $[1,18,30]$ with argon at higher density and somewhat lower confinement, growing during otherwise prolonged stationarity $\left(\approx 12 \tau_{\mathrm{E}}\right)$. The AP plasmas here seem also to gain from changes in the pedestal, however, as explained below.

Results for H-modes at $I_{\mathrm{p}}=2.5 \mathrm{MA}$ are summarized in figure 10(a), plotting confinement normalized to the ITERH97 scaling law [2] versus normalized density. One point is shown in the P (filled symbols) and one in the AP (open symbols) phases of each pulse with gas injection, choosing always steadiest conditions. Stationarity of septum AP data included is described further below. The outlined region depicts the extent of previous data from unseeded plasmas in the MkIIA/MkIIGB divertors at the same current of $2.5 \mathrm{MA}$, and clearly illustrates the usual $\mathrm{H}$-mode density limit owing to decaying confinement above $\approx 70 \%$ of the Greenwald density. Septum cases tend to lie towards the top of this earlier range around $f_{\mathrm{Gwd}} \approx 70-90 \%$ even with just $\mathrm{D}_{2}$ fuelling (black squares, triangles), and are further improved when impurities are injected (coloured squares, triangles). Their effect upon entering AP stages is portrayed by the dashed arrows, from which it is confirmed that without seeding confinement recovers by moving to lower density (black squares, triangles), but it does so for practically constant density in the presence of argon or/and neon (coloured squares, triangles). Hence the same good confinement is recovered but at higher density and very high combined values above the limit of previous data, up to $H_{97}=0.99$ at $f_{\mathrm{Gwd}}=0.94$ in figure $10(a)$, are achieved. Thus $H_{97} f_{\mathrm{Gwd}} \approx 0.93$, in terms of the so-called dimensionless figure-of-merit $[31,1,18]$, or normalized Lawson product, also often quoted. A progressive march to higher density while preserving confinement is clearly demonstrated by the sequence of septum APs (open squares) at $H_{97} \approx 1.05$, from the natural condition with no gas (grey), to deuterium only (black), to neon (red), to argon and argon plus neon (blue, mauve squares), i.e. heavier impurities seem to have the better effect. This species scaling is returned to later.

Cases at higher upper triangularity $\left(\delta^{\mathrm{u}} \approx 0.33\right.$ instead of 0.18 ) are also plotted (triangles), but little extra gain from such shaping is evident here. Impurity seeding may already be approaching the best performance presently attainable, therefore, pre-empting improvements separately established from strong shaping alone [32], i.e. these alternative schemes may be complementary ways to better conditions [1]. Counterpart divertor pulses with the strike-points in the MkIIGB corners, and on its vertical side targets, are similarly included (circles, circles with crosses). Lower densities obtained in the former configuration for similar inputs may partly reflect its maximized pumping capability, but there are also particularly strong changes in ELM properties from $\mathrm{P}$ to AP stages (see below). An especially large AP enhancement of refuelling and/or particle confinement is then observed, such that $f_{\mathrm{Gwd}}$ even increases after puffing ends. The same effect occurs to a lesser extent in the vertical targets cases as well, which tend to yield densities intermediate between those for 'corner' and septum plasmas. Comparing timeaveraged signals in a moving window of $0.5 \mathrm{~s} \sim \tau_{\mathrm{E}}$ for total $\mathrm{D} \alpha$ photon fluxes from the divertor and main torus, which are approximately proportional to respective source rates from majority ionization, shows divertor fuelling is indeed lower during puffing in the corner case, while torus refuelling is higher for vertical targets configuration. Otherwise, however, there are no significant differences between puffed cases with $\mathrm{D}_{2}$ only, neon, or argon, especially over the septum AP interval in which respective normalized densities are systematically raised. The key feature of restoring good confinement without lowering $f_{\mathrm{Gwd}}$ is thus not attributable simply to sources, but indeed involves more fundamental responses of particle transport to seeding effects equivalent to moderating the usual decline in H-mode fuelling efficiency. Pumping and perhaps threshold seem to be secondary factors.

All seeded septum instances in figure $10(a)$ have been selected according to a simple criterion to ensure greatest stationarity. The period $\Delta t$ for which $H_{97} f_{\mathrm{Gwd}}$ is continuously greater than or equal to $90 \%$ of its maximum value is divided by the mean energy confinement time throughout $\Delta t$, i.e. $\varsigma \equiv(\Delta t)^{2} / \int_{\Delta t} \tau_{\mathrm{E}} \mathrm{d} t$. This number $\varsigma$ is plotted for all seeded septum APs examined in figure 10(b), encompassing impurity injection just during the $\mathrm{P}$, or also into the AP stage (e.g. as for shot \#50350 in figure 9), plus optimization of short, low 'blips' of argon and deuterium during the AP. The small difference between respective figure-of-merit values affirms these extra, low inputs can be incorporated without adversely affecting performance, but their success in lengthening the stationary phase of highest confinement, density and radiation is apparent. Superior properties $H_{97} f_{\mathrm{Gwd}} \geqslant 0.8$ are sustained for up to ten confinement times, and later experiments have continued to develop this further $[1,18]$. Seeded septum plasmas included in figure 10(a) (and figure 11) have accordingly been restricted just to steadier results having $\varsigma \geqslant 4$. Such beneficial AP adaptations emphasize that the strategy under investigation is actually one of optimized fuelling and radiation, rather than simply an unpuffed regime. It again complements separate experiments on long timescale density increases in JET [33], but through seeding offers higher radiation fractions as well.

A crucial aspect already noted is that higher $f_{\text {rad }}$ is achieved without significant increase of $Z_{\text {eff }}$. A summary for the same 2.5 MA dataset as in figure $10(a)$, in particular including seeded septum cases only with $\zeta \geqslant 4$, is given in figure 11. The trend for seeded APs (coloured open symbols) to increase $f_{\text {rad }}$ while retaining similar purity to their unseeded counterparts (grey, black open symbols) is confirmed; furthermore, their absolute $Z_{\text {eff }}$ values are reasonably low $(\leqslant 2.5)$ even when impurities are infused into the AP stages themselves (blue, mauve open symbols). In fact, AP blips specifically of deuterium generally help not only to extend stationarity but also to keep down line-average $Z_{\text {eff }}$, and intrinsic carbon impurity content was noted to be lowered in at least one AP supplied with a more continuous trickle of $\mathrm{D}_{2}$ (cf figure $10(b)$ ). On the other hand, this is partially offset by a corresponding tendency to depress $f_{\text {rad }}$, back to levels no higher than those obtained without any AP additional dosing at all. Values of $Z_{\text {eff }}$ and $P_{\text {rad }}$ produced remain precisely in agreement with the Matthews scaling 


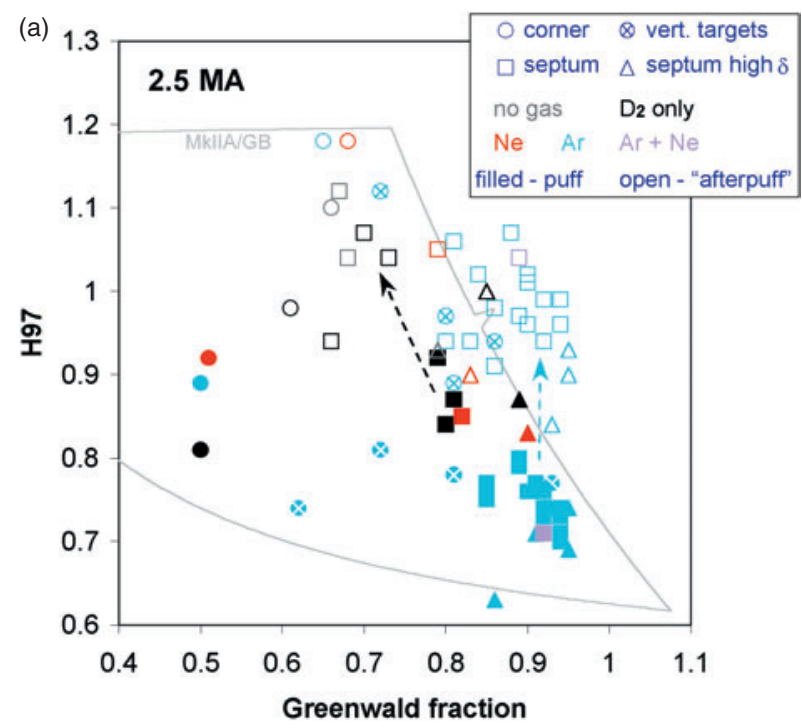

(b)

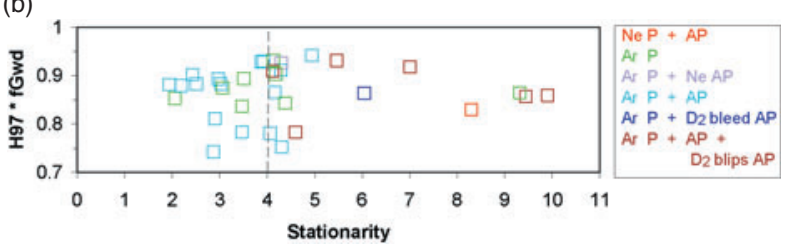

Figure 10. (a) Normalized confinement against Greenwald density fraction for JET ELMy H-modes at 2.5 MA, plotting one point during main puffing (filled symbols) plus one point during the AP (open symbols) for each discharge. Different configurations, fuelling and seeding are discriminated. Septum plasmas generally have the highest performance, but without impurities recover better confinement by moving to lower density in the AP (black dotted arrow). When seeded, high density is sustained into the AP as well, resulting in outstanding combined values (blue dotted arrow). A progressive march to higher density at $H_{97} \approx 1.05$ is indicated for increasingly heavy seed species (respective open squares). Outlined is the extent of previous unseeded data at the same current in MkIIA/MkIIGB divertors, with the conventional H-mode density limit due to falling confinement evident for $f_{\mathrm{Gwd}} \geqslant 0.73$. All data points shown are chosen during steady phases, with the stationarity particularly of septum APs being elaborated in $(b)$. (b) AP performance of septum discharges at 2.5 MA with seeding in main puff $(\mathrm{P})$ stage, then optimizing extra impurity and $\mathrm{D}_{2}$ fine inputs in AP stage. Stationarity is estimated by the time for which $H_{97} f_{\mathrm{Gwd}}$ is continuously $\geqslant 90 \%$ of its maximum value, normalized by mean $\tau_{\mathrm{E}}$ over this interval. Extra, low sources in the AP extend its high-performance phase without significantly degrading its level. Respective points included in figures $10(a)$ and 11 are restricted to those with stationarity $\geqslant 4$.

[4] during septum $P$ phases, which of course reproduce the same type of experiment as its original basis. However, owing to its dependence $\propto 1 / \bar{n}_{\mathrm{e}}^{2}$, the scaling consistently underestimates effective charge by $\approx 30 \%$ during subsequent APs, emphasizing the higher densities then reached, i.e. the scaling is less well adapted to these new states. It is also evident in figure 11 that similar $Z_{\text {eff }}$ is obtained for both diverted and septum unseeded APs (grey, black open symbols), recalling a weak effect of increasingly closed divertor designs on intrinsic impurity content of JET plasmas described before [16, 34, 35]. During deuterium-only puffing (black filled symbols) or with seeding (coloured symbols), though, $Z_{\text {eff }}$ tends to be higher in diverted cases, particularly the most strongly pumped corner

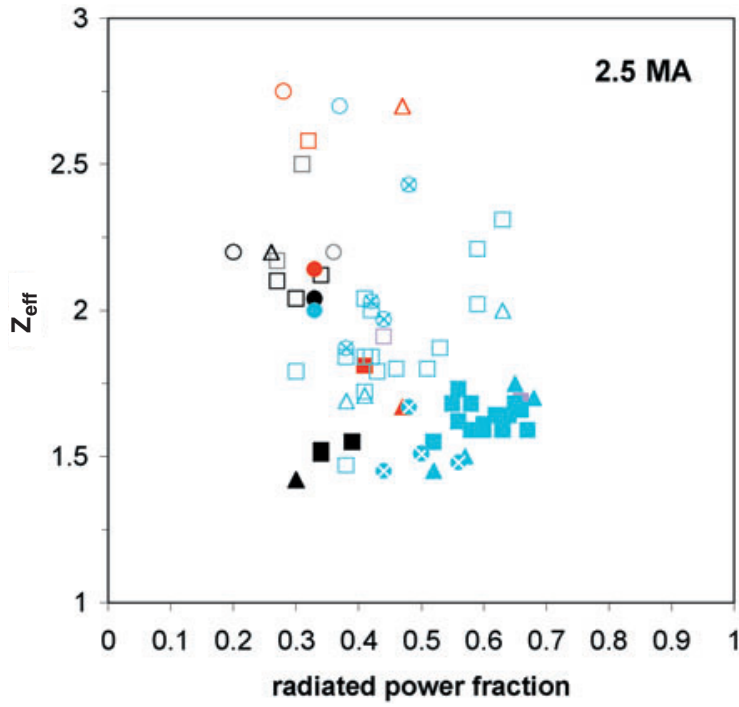

Figure 11. Central line-average $Z_{\text {eff }}$ against radiated power fraction $f_{\text {rad }}$ for the same dataset (also same symbol key) as in figure 10(a). Particularly for septum APs with argon, $Z_{\text {eff }}$ stays similar to or lower than in unseeded counterparts, while $f_{\text {rad }}$ is raised. Note such APs with prolonged stationarity through additional 'blips' of $\mathrm{D}_{2}$ and argon (cf figure 10(b)) actually have the lower $f_{\text {rad }}$ values.

configuration (circles), in accordance with their lower densities already mentioned (cf figure 10(a)). Extrinsic impurities are therefore better controlled and provide a greater radiation fraction at higher density, realized here in septum geometry. Its associated $f_{\text {rad }}$ in turn is higher, reaching up to $\approx 60 \%$, and $Z_{\text {eff }}$ lower, using argon rather than neon (blue symbols versus red).

The crux for seed impurities is whether they are contained within the plasma periphery, as realized in RI-modes on TEXTOR-94 [7], or whether they penetrate adversely throughout its volume. Integrating over tomographic reconstructions from bolometry, the fractions of power radiated in the divertor and main plasma above the X-point during a subset of APs are plotted in figure 12(a). The fraction from and below the X-point appears to change little when impurities are injected, and unsurprisingly is higher in the corner diverted arrangement. For septum geometry, though, a substantial increase of the main torus fraction accompanies particularly argon, as opposed to neon, seeding. Hence more total radiation is produced for a given $Z_{\text {eff }}$ from argon than neon (figure 11), as had been found previously [17], and in septum cases more of its emission is induced in the main plasma instead of the divertor region, as required to form a radiating mantle. Estimated one-dimensional radiation profiles from tomographic inversion of bolometer signals are shown during one such septum AP (\#50476) with argon $(\mathrm{P}+\mathrm{AP})$ in figure $12(b)$, from which it emerges that initially $(<21.4 \mathrm{~s})$ much of the enhanced bulk radiation is indeed concentrated at the edge. Its inward spread and inexorable rise in the core subsequently ( $>21.4 \mathrm{~s}$ ), suggestive of central impurity accumulation, actually seems to be correlated with disappearance of sawteeth, as elaborated elsewhere [36]. In conjunction, though, roll-over of confinement and central electron temperature around $21 \mathrm{~s}$ in this pulse (see bottom panel in figure 15) precede onset of deep impurity penetration, so some other alteration of plasma conditions could be involved 

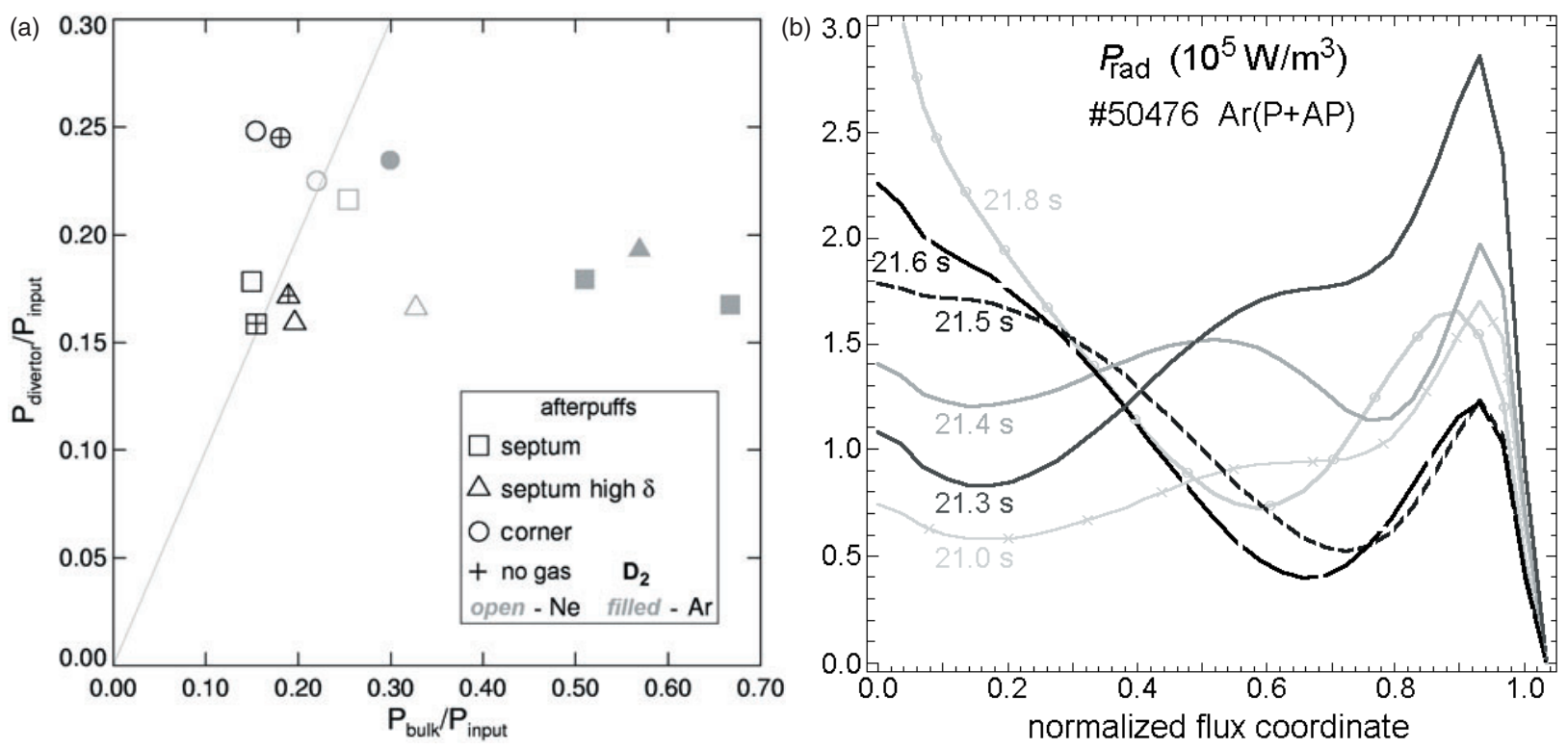

Figure 12. (a) Fractions of power radiated from the divertor plus X-point, versus main plasma, regions, using tomographic reconstruction of bolometry for a subset of APs at 2.5 MA. Septum APs with argon display a large increase of radiation in the main torus, as required to form a mantle. (b) Radial profiles of radiated power density from tomographic inversion of bolometry for a septum AP with argon. Times specified span an ELM-free interval and show peaking initially in the edge, corresponding to a mantle. Subsequent central peaking starts when sawteeth are lost, after roll-over of confinement.

too. Details of seed impurity transport in such cases are examined further in [37]. These advantages of argon over neon therefore recall the favourable scaling with heavier impurity ions noted in figure 10(a) before, i.e. a given proportion of impurity is not only less diluting for given $Z_{\text {eff }}$, but seems more effective at radiating and assisting $\mathrm{H}$-mode performance in JET, employing the heavier ion species. Conversely in DIII-D, neon was found to induce largest improvements $[13,38]$, although these results applied to L-mode plasmas. A species scaling for seeding best adapted to plasma parameters of a given device is consequently implied.

\subsection{ELMs and pedestal values}

As stated at the outset, a prime motive behind intentional seeding of $\mathrm{H}$-modes with radiating species is to try to lessen intense intermittent loads on surfaces caused by their ELMs, without simultaneously degrading confinement. On JET, both $\mathrm{D}_{2}$ and especially impurity puffing have a pronounced impact on usual Type I ELMs for a well confined H-mode, recovering a recognized $[3,4]$ decrease of their amplitudes and increase of their frequency $v_{\mathrm{ELM}}$ to something resembling Type III ranges [17,39] but of course for degraded confinement [3]. This compression of ELMs becomes stronger with heavier seed species. Marked effects persist into AP stages too, where conversely the frequency of ELMs tends steadily to be reduced with increasingly potent seeding [39] (see D $\alpha$ signals indicated in figure 15; similar behaviour has been reported on JT-60U [9]). Complex compound signatures of no unambiguous frequency are also commonly produced. Using argon eventually extended ELM-free phases, sometimes ending in large ELMs, and typically with intervening bursts of grassy fluctuations, are reached. As just mentioned above, confinement tends to peak and then decline again in long such ELM-free intervals, while total radiated power rises monotonically until dropping again at its succeeding ELM. Recall in the example given, however, that it builds up mostly at the edge until sawteeth are lost, after confinement has already rolled over. The trend to lower $v_{\mathrm{ELM}}$ in APs is summarized for a subset mainly of septum plasmas in figure 13, eliminating instances with compound ELMs, i.e. the figure concentrates on the clearer type of response, although more complicated behaviour does also occur. Those ELMs depicted slow considerably from $\mathrm{P}$ to AP stages, especially with argon, and moreover largely in agreement still with the representative so-called Fishpool scaling of normalized confinement against $v_{\text {ELM }}$ deduced previously for JET unseeded discharges in the MkIIA/AP divertors [40]. Such conformity suggests that changes in the underlying pedestal energy may be similar as well, though the key point to recall is that new impurity seeded results are exhibiting these comparable confinement and pedestal values both for higher radiated power levels and especially for higher fractions of the Greenwald density. Most conspicuous departures from Fishpool predictions, extrapolated below their original lower frequency range [40], occur at very low $v_{\mathrm{ELM}}$ with argon injection (open blue symbols), so hinting at almost constant AP confinement factor below $\approx 20 \mathrm{~Hz}$ (open squares, triangles).

Assuming small corrections for fast particles, electron pedestal properties may be estimated from an outer interferometer channel viewing along a major radius of $3.75 \mathrm{~m}$, together with electron temperature at the point of gradient change in profiles from edge ECE measurements [3]. Again averaging signals in a moving $0.5 \mathrm{~s}$ interval to smooth over ELMs, quantities for a septum sequence from natural, to pure $\mathrm{D}_{2}$, to neon and increasing argon inputs are traced in figure 14 . Temperature $\left\langle T_{\mathrm{e}}^{\text {ped }}\right\rangle_{t}$ is respectively more strongly depressed during puffing, and recovers proportionally in the AP such that the neon-seeded plasma remains below reference cases, while argon seeding yields lower values still. Maintenance of higher 
$f_{\text {Gwd }}$ with impurities, however, is supported by an elevated pedestal density $\left\langle n_{\mathrm{e}}^{\text {ped }}\right\rangle_{t}$ from the main P stage being held at almost the same level into the AP. In the example with neon this results in a significantly increased electron pedestal pressure $\left\langle p_{\mathrm{e}}^{\text {ped }}\right\rangle_{t}$ for almost identical total stored energy $\left\langle W_{\mathrm{dia}}\right\rangle_{t}$ as in the unseeded APs, suggesting a partial loss of core confinement. Recall this species also leads to relatively lower $f_{\text {rad }}$ and higher $Z_{\text {eff }}$ (figure 11). With argon, in contrast, the reference diamagnetic energy $\left\langle W_{\text {dia }}\right\rangle_{t}$ is recovered in the AP for a slightly reduced $\left\langle p_{\mathrm{e}}^{\text {ped }}\right\rangle_{t}$, hinting that now greater energy is stored in the core on top of the pedestal. Lower time-averaged $\left\langle p_{\mathrm{e}}^{\text {ped }}\right\rangle_{t}$ is

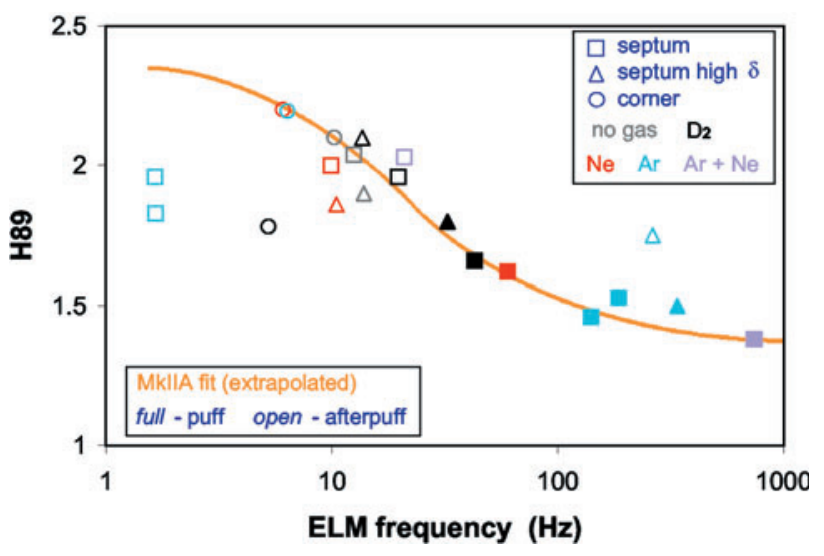

Figure 13. Normalized confinement against ELM frequency for a subset of plasmas exhibiting clearly defined ELMs in both P and AP stages, i.e. alternative compound ELM results are excluded. At least in septum geometry, ELM frequency always falls from $\mathrm{P}$ to AP with or without impurities, but with seeding it is raised in the former and then yet further reduced in the latter. Superimposed ( - ) is a downward extrapolation of the so-called Fishpool relation derived previously for unseeded states in the MkIIA/AP divertors [40]. obtained despite lower frequency ELMs too, actually counter to previous experience embodied in the Fishpool relation [40], but consistent with increased edge radiation. While not yet accounting for the accompanying ion response, these features therefore tentatively indicate improved core confinement due to argon injection, according with an incipient RI effect, just as being sought. Its occurrence has been confirmed in TRANSP calculations [41] for similar septum APs, which show that argon seeding reduces the effective thermal diffusivity over most of the plasma cross-section. Again similar gains in core confinement following argon injection have also been found on JT-60U $[9,42]$.

A next-step device will probably need to handle largest ELM effluxes within their natural variability rather than simply their average size [4], so that amplitudes in terms of energy expelled each time $(\Delta W / W)$ must be mitigated even ahead of their frequencies. Unsmoothed signals of total stored energy are correlated with $\mathrm{D} \alpha$ emission in figure 15, still for the foregoing sequence on the septum, plus the pulse in figure $12(b)$ with yet higher argon input. Short $(0.8 \mathrm{~s})$ time windows of fastest sampling rate, shown at $5 \mathrm{kHz}$, are also included. In fact, though ELMs are clearly slowed by mounting impurity seeding, there is no evidence that their amplitudes are being altered from about $(\Delta W / W) \approx 7-8 \%$ throughout, until they are finally quenched in the last case (\#50476) where conditions are not sustained, as already described. Comparable fixed ELM sizes for decreasing frequency with argon seeding have been detected on JT-60U [9], although a more stable edge regime may moderate its fluctuations at higher density [42] $\left(f_{\text {Gwd }} \approx 0.7\right)$. Fixed amplitudes would accord with nearly constant pedestal pressure in JET cases, just disclosed, together with a ballooning mode picture of ELM instabilities [43], if the pedestal width were also unchanged. An alternative dependence of ELM size on
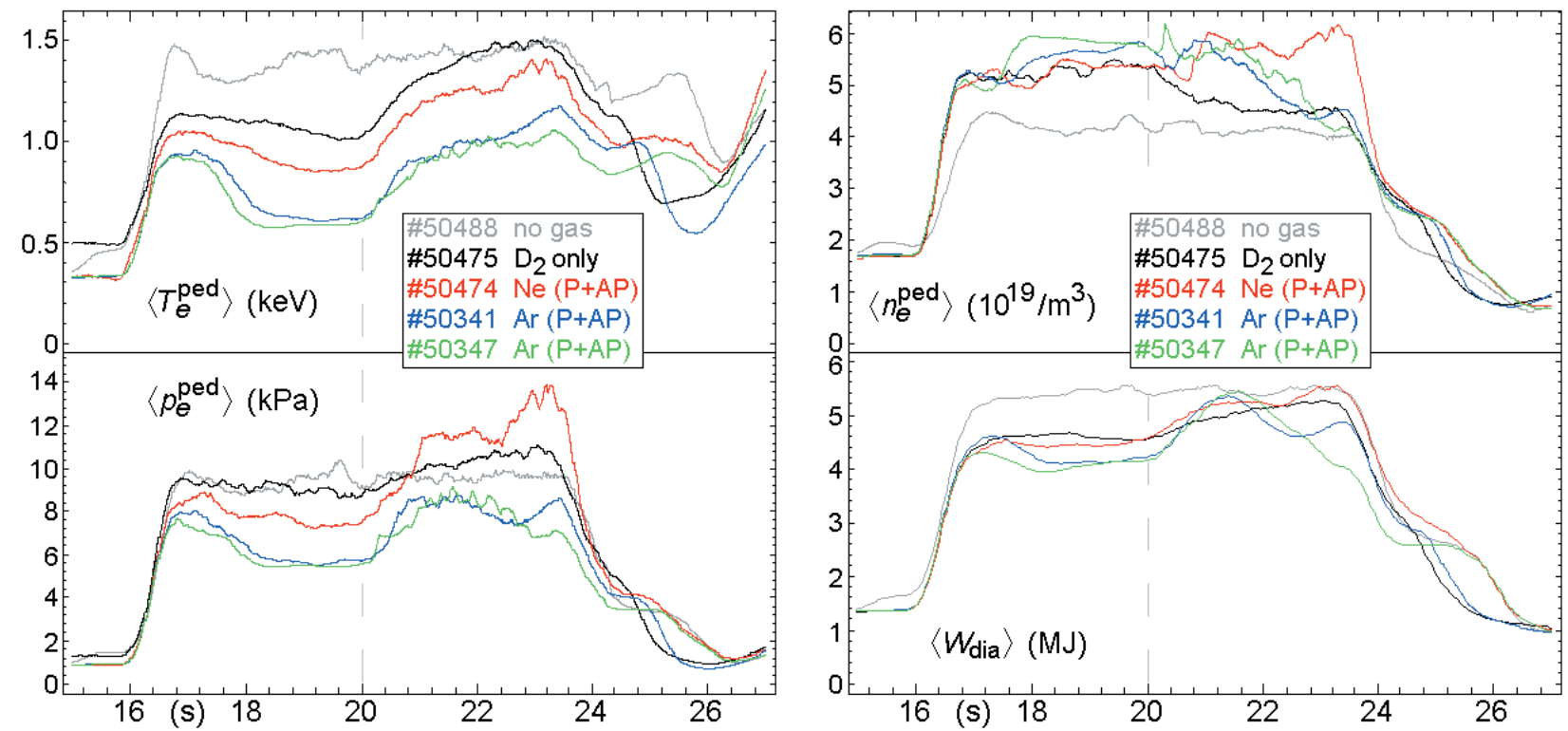

Figure 14. Time-averaged signals in a moving $0.5 \mathrm{~s}$ window of electron pedestal temperature from edge ECE, pedestal density from an interferometer channel at $3.75 \mathrm{~m}$, electron pedestal pressure, and diamagnetic stored energy assuming negligible fast particle contributions. A sequence of septum plasmas with varying seeding is depicted, each AP stage beginning at $20 \mathrm{~s}$ (vertical - - -). Note $\left\langle T_{\mathrm{e}}^{\text {ped }}\right\rangle_{t},\left\langle p_{\mathrm{e}}^{\text {ped }}\right\rangle_{t}$ and $\left\langle W_{\text {dia }}\right\rangle_{t}$ always rise from the $\mathrm{P}$ to AP, whether seeded or not. Injecting impurities also maintains higher $f_{\mathrm{Gwd}}$ into the AP, at least partly by keeping higher $\left\langle n_{\mathrm{e}}^{\text {ped }}\right\rangle_{t}$. 


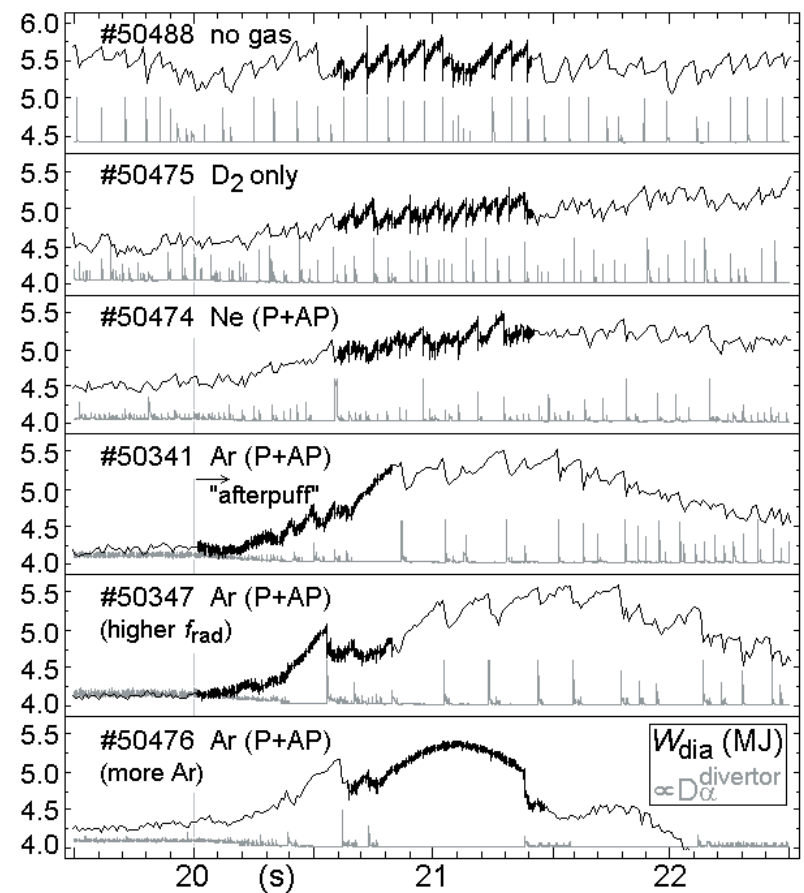

Figure 15. Unsmoothed total stored energy data correlated with divertor $\mathrm{D} \alpha$ signals for the same sequence of septum APs as in figure 14, plus that with even stronger argon seeding from figure $12(b)$. Periods of faster magnetic data sampling are also included. ELMs appear to be slowed by seeding but not obviously lessened in size, until stopped.

electron collisionality in the pedestal which has recently been recognized [44] will be discussed separately [45]. Another aspect still to be determined, on the other hand, is how much of the plasma energy $(\Delta W / W)$ ejected is then deposited in concentrated target loads, since some of it at least could of course be dispersed in the edge by heightened radiation due to seeding. First JET use of calibrated infrared camera data to study this question is addressed in [39]. A final issue exposed by ELM adjustments and which remains under examination concerns magnetic fluctuations detected during APs. Mode analyses of cross-correlated phase differences between fast magnetic signals at two toroidal locations [46] reveal broadband activity $(\leqslant 80 \mathrm{kHz})$ rotating in the electron-drift direction throughout best confinement phases [12]. Equivalent effects in the ion drift direction are not seen, except perhaps after reversion to L-mode [46]. The electron-drift features are interrupted by large ELMs or suppressed by intervals of small, fast ELMs, and most interestingly have been noticed to die gradually away during an ELM-free period in one seeded septum plasma, like that for pulse \#50476 in figure 15, as normalized confinement rolls over [12]. These modes are difficult to locate radially, and to relate quantitatively to transport, but seem to recall the respective roles of electron and ion turbulence in the transition to improved RI from L-mode confinement introduced before [24].

\section{Summary and discussion}

Initial studies of the scaling of RI regimes to JET have been made by injecting medium- $Z$ impurities into a range of different plasma conditions and configurations. While the definitive improvements of RI-mode as established on TEXTOR-94 $[6,7,19,21]$ have not yet fully emerged in JET, considerable benefits still have been found.

- The discovery conditions of TEXTOR-94 [6,7] have been most closely reproduced in JET limiter L-modes seeded with neon, but rapid penetration into the plasma core and formation of MARFEs both seem to prevail. Crucially, distinct particle transport accompanying lower collisionality to that in TEXTOR-94 is inferred. Without much stronger interior particle sources this prevents access to greater central peaking of the density, presently thought to underlie strong stabilization of ITG modes in RI transitions [24].

- Alternatively in divertor L-modes with neon seeding, transient increases of normalized confinement up to $H_{89} \approx 2$ for more moderate radiation and Greenwald fractions $f_{\text {rad }}, f_{\text {Gwd }} \approx 40 \%$ have been obtained, closely resembling related results in DIII-D [13]. In JET, however, the rise of radiated power with seeding is relatively much slower, so that comparable confinement and density are attained for lower $f_{\text {rad }}$. Neon also tends to permeate rapidly into the core again. Local transport analyses with TRANSP find ion thermal diffusivity is reduced across the whole plasma, though competing electron instabilities may contribute to a more modest impact on confinement. A doubling of central ion temperature and thermal neutron production rate has been observed in latest results [26]. Further optimization of the current density profile is required more successfully to avoid limiting MHD instabilities [26]. Next experimental aims will include better control of added impurities, plus resolution of the roles of increased $Z_{\text {eff }}$, density peaking and/or toroidal rotational shear during turbulence reduction.

- Most notably, for impurity injection into ELMy H-modes at low triangularity, long 'AP' phases following main gas fuelling have demonstrated a major gain in terms of integrated performance, which will be essential to operation of a next-step device. These phases always recover high confinement, but when seeded retain high densities close to the Greenwald level as well. Particularly with argon seeding of discharges formed on the MkIIGB divertor septum, outstanding combined properties up to $H_{97}=0.99$ at $f_{\mathrm{Gwd}}=0.94$ have been achieved in campaigns during 2000, exceeding the conventional $\mathrm{H}$-mode density limit for divertor geometry. Further work in 2001 is presented in $[1,18]$. Stationary duration of good conditions has been prolonged for many confinement times by tailoring low, supplementary spurts of deuterium and argon after the end of main puffing. How the usual degradation of fuelling efficiency in the density limit is tempered remains to be identified, majority sources being roughly unchanged, but in this regard impurity seeding thus forms part of the wider development of optimized fuelling schemes [33] again vital for very long next-step pulses. Adding impurities also increases the fraction of exhaust power dispersed as radiation, and values in septum APs up to $f_{\text {rad }} \approx 60 \%$ have been reached for almost no deterioration in $Z_{\text {eff }}$. Effects become stronger with heavier seed species in JET, both higher performance and more 
radiation for given $Z_{\text {eff }}$ arising with argon than with neon. Contrasting this with the greater effectiveness of neon $[13,38]$ in DIII-D therefore suggests a scaling of the most suitable seed species with the plasma parameters of each device. Furthermore, in JET septum cases argon emission stays more concentrated within the plasma periphery as required to form a mantle, at least perhaps until sawteeth are lost. Efforts will be continued in next experiments to test maintenance of sawteeth with central ICRH [36], since it could then provide for long steady phases with sustained edge retention of impurities. Argon seeding tends to lower ELM frequencies in the AP stage too, even though electron pedestal pressure and fractional energy drops $(\Delta W / W)$ seem only slightly changed from unseeded behaviour. High pedestal density from the $\mathrm{P}$ is kept, and seems partly to support the higher global density, in the AP, although evidence of greater central peaking has also been seen $[1,16,30]$. Further exploration will next be undertaken of the tentative RI-like improvement of core confinement so implied, and how far actual target loads due to ELMs might still be abated by heightened radiation. In particular, attention needs to be given to similar enhanced $\mathrm{H}$-mode states with seeding, and greater central peaking of plasma density, in divertor geometry. This will clarify the comparison with other strong shaping studies [32], while focusing on the situation for ITER.

Each of these H-mode advances in terms of integrated properties, stationarity and radiated power fraction moves precisely in the direction required for standard operation in a burning next-step tokamak. In addition to high performance, ITER must have a viable approach to handling its particle and especially its power exhaust, which is expected to rely upon a large fraction of radiation in order to secure endurable divertor loads. A radiating mantle as intended by seeding experiments is consequently a necessity under next-step conditions and coincident gains in terms of performance and steadiness emerging in JET reinforce its usefulness. For ITER, injection of krypton is proposed to increase radiation, so improvement of seeding effects and emitted fractions with heavier ions reported here again seem encouraging results. Thus, impurity seeding may become proportionally more beneficial in the context of a scaled-up device and species. A prominent issue remaining concerns ELMs, which would extrapolate from typical Type I fluctuations in JET to extremely severe transients in ITER. Impurity injection appears so far mainly to reduce their frequency in APs rather than their amplitude, consistent with the pedestal being practically unaffected, while confinement seems adversely to roll-over when they are stopped altogether. Controlling ELM energies and resulting surface loads is therefore a key topic which strategies such as plasma shaping and impurity seeding will continue to address.

\section{Acknowledgments}

An earlier preview of part of these results was first presented at the 18th IAEA Fusion Energy Conference, Sorrento, Italy, October 2000. Work has been carried out by wide international collaboration initiated during the Joint Undertaking in 1999, but most extensively conducted under the
EFDA-JET arrangements from 2000 onwards. Participation of UKAEA authors is supported jointly by the UK Department of Trade and Industry and Euratom.

\section{References}

[1] Ongena J et al 2001 Plasma Phys. Control. Fusion 43 A11

[2] Cordey J G et al 1997 Plasma Phys. Control. Fusion 39 B115

[3] Saibene G et al 1999 Nucl. Fusion 391133

[4] Matthews G F et al 1999 Nucl. Fusion 3919

[5] Kallenbach A et al 1995 Proc. 22nd EPS Conf. on Controlled Fusion and Plasma Physics (Bournemouth, July 1995) vol 19C (ECA) II-005

[6] Messiaen A M et al 1994 Nucl. Fusion 34825

[7] Messiaen A M et al 1996 Phys. Rev. Lett. 772487

[8] Lazarus E A et al 1984 J. Nucl. Mater 12161

[9] Kubo H et al 2001 Nucl. Fusion 41227

[10] Maddison G P et al 2000 RI-mode experiments JET report Descriptive analysis of 1999 Task Force P data, JET-R(00)02 (July 2000) ch 10

[11] Unterberg B et al 2000 Proc. 27th EPS Conf. on Controlled Fusion and Plasma Physics (Budapest, June 2000) vol 24B (ECA) p 1408 CD-ROM file P4_038

[12] Maddison G P et al 2001 Proc. 18th Int. Conf. on Fusion Energy 2000 (Sorrento, October 2000) (Vienna: IAEA) CD-ROM file EX5_4 and http://www.iaea.org/programmes/ripc/ physics/fec2000/html/node1.htm

[13] McKee G R et al 2000 Phys. Plasmas 71870

[14] Jackson G L et al 2000 Proc. 27th EPS Conf. on Controlled Fusion and Plasma Physics (Budapest, June 2000) vol 24B (ECA) p 241 CD-ROM file P1_047

[15] Jackson G L et al 2000 Use of impurity injection for improved performance in the DIII-D and JET tokamaks 42nd APS DPP Meeting (Quebec City, October 2000) HP1-057 and http://web.gat.com/pubs-ext/APS00/Jackson_pos.pdf

[16] JET Team (presented by R D Monk) 1999 Nucl. Fusion 39 1751

[17] Strachan J D et al 2000 Plasma Phys. Control. Fusion 42 A81

[18] Dumortier P et al 2002 Plasma Phys. Control. Fusion 44 1845

[19] Unterberg B et al 2001 Proc. 18th Int. Conf. on Fusion Energy 2000 (Sorrento, October 2000) (Vienna: IAEA) CD-ROM file EX5_2 and http://www.iaea.org/programmes/ripc/physics/ fec2000/html/node1.htm

[20] Yushmanov P N et al 1990 Nucl. Fusion 301999

[21] Ongena J et al 1999 Plasma Phys. Control. Fusion 41 A379

[22] Tokar M Z et al 1995 Phys. Scr. 51665

[23] Telesca G et al 2000 Nucl. Fusion 401845

[24] Tokar M Z et al 2000 Phys. Rev. Lett. 84895

[25] Tokar M Z et al 2002 Plasma Phys. Control. Fusion 441903

[26] Jackson G L et al 2002 Plasma Phys. Control. Fusion 441893

[27] Murakami M et al 2001 Nucl. Fusion 41317

[28] Tokar M Z et al 1999 Plasma Phys. Control. Fusion 41 B317

[29] Horton L D et al 1999 Proc. 26th EPS Conf. on Controlled Fusion and Plasma Physics (Maastricht, June 1999) vol 23J (ECA) p 193 CD-ROM file P1021

[30] Suttrop W et al 2002 Phys. Plasmas 92103

[31] Horton L D et al 1999 Nucl. Fusion 391

[32] Kallenbach A et al 2002 Nucl. Fusion 421184

[33] Valovic M et al 2002 Plasma Phys. Control. Fusion 441911

[34] McCracken G M et al 1999 Nucl. Fusion 3941

[35] Guo H Y et al 2000 Nucl. Fusion 40379

[36] Nave M F F et al 2001 Proc. 28th EPS Conf. on Controlled Fusion Plasma Physics (Madeira, June 2001) vol 25A (ECA) p 961 CD-ROM file P3.009

[37] Puiatti M E et al 2002 Plasma. Phys. Control. Fusion 44 1863 
[38] Murakami M et al 2000 Proc. 27th EPS Conf. on Control. Fusion and Plasma Physics (Budapest, June 2000) vol 24B (ECA) p 560 CD-ROM file P2_026

[39] Jachmich S et al 2002 Plasma Phys. Control. Fusion 441879

[40] Fishpool G M 1998 Nucl. Fusion 381373

[41] Ongena J et al 2001 Phys. Plasmas 82188

[42] Kubo H et al 2002 Phys. Plasmas 92127

[43] Connor J W et al 1998 Phys. Plasma 52687
[44] Loarte A et al 2001 Proc 18th Int Conf. on Fusion Energy 2000 (Sorrento, October 2000) (Vienna: IAEA) CD-ROM file ITERP_11 and http://www.iaea.org/programmes/ripc/ physics/fec2000/html/node1.htm

[45] Maddison G P and Ongena J Improved ELM scaling with impurity seeding in JET, in preparation

[46] Sharapov S 2000 EURATOM/UKAEA Fusion Association Private communication 